\title{
MEDIDA DAS \\ DESIGUALDADES DE APRENDIZADO ENTRE ESTUDANTES DE ENSINO FUNDAMENTAL
}

JOSÉ FRANCISCO SOARES VICTOR MAIA SENNA DELGADO

\section{RESUMO}

Neste artigo, uma situação de igualdade educacional é definida como aquela em que quaisquer grupos de estudantes têm a mesma distribuição de desempenho cujos valores correspondem a aprendizados que os habilitam a uma inserção produtiva $e$ pessoalmente satisfatória na sociedade. 0 principal objetivo desse texto é introduzir um indicador de desigualdade educacional definido como a distância entre a distribuição ideal de desempenho e a observada em um dado grupo de estudantes. As análises apresentadas mostram que as desigualdades no ensino fundamental brasileiro são muito grandes e que nunca desaparecerão caso as melhorias continuem no ritmo atual.

PALAVRAS-CHAVE INDICADORES EDUCACIONAIS - DESIGUALDADES EDUCACIONAIS • PROVA BRASIL・ENSINO FUNDAMENTAL. 


\section{MEDICIÓN DE LAS DESIGUALDADES DE APRENDIZAJE ENTRE ESTUDIANTES DE LA EDUCACIÓN BÁSICA \\ RESUMEN}

En este artículo se define una situación de igualdad educacional como aquella en la que cualesquiera grupos de estudiantes tienen la misma distribución de desempeño cuyos valores corresponden a aprendizajes que los habilitan a una inserción productiva y personalmente satisfactoria en la sociedad. El principal objetivo de este texto es el de introducir un indicador de desigualdad educacional definido como la distancia entre la distribución ideal de desempeño y la observada en un determinado grupo de estudiantes. Los análisis presentados muestran que las desigualdades en la educación básica brasileña son muy grandes y que nunca desaparecerán si las mejoras sigan ocurriendo en el actual ritmo.

PALABRAS CLAVE INDICADORES EDUCACIONALES - DESIGUALDADES EDUCACIONALES・PROVA BRASIL・EDUCACIÓN BÁSICA.

\section{ASSESSMENT OF LEARNING INEQUALITIES AMONG ELEMENTARY SCHOOL STUDENTS}

ABSTRACT

Educational equality, as defined in this article, is a condition in which any group of students presents the same performance distribution whose values match the learning experiences that enable them to have a productive and personally satisfying insertion into society. The main objective of this article is to introduce an indicator of educational inequality defined as the gap between the ideal performance distribution and that observed in a given group of students. The analyses presented here show that inequality in Brazilian elementary schools is very large and will never disappear if the improvements continue at the current pace.

KEYWORDS EDUCATIONAL INDICATORS • EDUCATIONAL INEQUALITIES • PROVA BRASIL・BASIC EDUCATION. 
1 O Saeb tem três componentes: a Avaliação Nacional da Educação Básica, a Avaliação Nacional do Rendimento Escolar (Prova Brasil) e a Avaliação Nacional da Alfabetização (ANA). Nesse texto, usam-se os dados da Prova Brasil para o ensino fundamental. A descrição completa dessas avaliações está disponível em: $<$ http://portal.inep.gov.br/web/saeb/ aneb-e-anresc>. Acesso em: nov. 2016

\section{INTRODUÇÃO}

O artigo 205 da Constituição Federal Brasileira (BRASIL, 2004, p. 121) estabelece que o direito à educação é assegurado quando são adquiridos os aprendizados necessários para se atingir "o pleno desenvolvimento da pessoa, seu preparo para o exercício da cidadania e sua qualificação para o trabalho". Constitucionalmente, portanto, o direito à educação é o direito de aprender. Para verificar se esse direito foi garantido a todos os cidadãos, principalmente aqueles na idade de escolaridade compulsória, o governo federal instituiu o Sistema de Avaliação da Educação Básica (Saeb) que tem, concomitantemente, outras funções pedagógicas e escolares. ${ }^{1}$

As medidas de aprendizado produzidas pelo Saeb têm mostrado que o direito à educação não está garantido para todos. Há muitos estudantes que, embora matriculados em uma escola de ensino fundamental, não aprendem o necessário para suas vidas e existem grandes diferenças de aprendizado entre grupos de alunos, definidos por critérios sociodemográficos, como gênero, cor-raça, nível socioeconômico (NSE) e região de residência. Ou seja, o ensino 
fundamental brasileiro tem grandes e profundos problemas de qualidade e de desigualdade.

O nível do aprendizado dos estudantes domina o debate sobre a qualidade da educação enquanto a desigualdade ou fica em segundo plano ou é completamente ignorada. Contribui para isso o fato de que, como há um indicador amplamente usado que informa adequadamente sobre o aprendizado - o Índice de Desenvolvimento da Educação Básica (Ideb) -, não há um indicador padrão para a desigualdade educacional. Introduzir um indicador que possa cumprir essa função é o principal objetivo deste artigo.

Em 1979, Amartya Sen (1979) sugeriu que, antes de se discutir a desigualdade, é necessário dar resposta objetiva à pergunta “igualdade de quê?". Sua reflexão sobre esse tema impactou todos os debates posteriores sobre desigualdade. Assim, para tratar das desigualdades educacionais é preciso estabelecer o sentido preciso a ser adotado para o conceito de igualdade educacional.

Nos estudos sobre desigualdade de renda toma-se como ideal a situação em que todos os indivíduos de uma dada população têm a mesma renda fixada como renda média obtida pela divisão equitativa de toda a renda de um país. O coeficiente de Gini, indicador padrão de desigualdade de renda, mede quão longe da situação ideal a situação real está. O conceito de igualdade educacional não pode ser definido como mera translação para a situação educacional desses conceitos. Isso porque os estudantes são naturalmente diferentes, têm vontades e projetos de vida diferentes. Assim sendo, diferenças de aprendizado entre indivíduos específicos podem ser fruto apenas de variações naturais ou de escolhas pessoais livres e informadas, não constituindo, portanto, evidência de desigualdade educacional.

A igualdade educacional é categoria essencial no debate educacional quando as unidades de análise são grupos de alunos. Isso exige que o estudo do desempenho de um grupo de estudantes deva ser feito usando-se a distribuição das proficiências de todos os estudantes do grupo. ${ }^{2}$ Em termos de aprendizado, a situação ideal é descrita por meio da distribuição das medidas de aprendizado que assume valores
2 ○ termo "distribuição" é usado aqui no sentido estatístico, ou seja especificação dos valores que a medida de aprendizado de cada aluno pode assumir e da frequência de ocorrência de cada um desses valores. 
associados aos aprendizados necessários na vida dos estudantes e tem variação compatível com a diversidade sem exclusão.

Assume-se, neste artigo, que a situação de igualdade em educação ocorre quando a distribuição dos aprendizados dos estudantes, pertencentes a quaisquer grupos, é igual à distribuição de referência. Diante disso, a medida de qualidade da educação deve ser a distância da distribuição real observada até a distribuição de referência e, consequentemente, a desigualdade é estudada comparando-se o valor dessa distância para diferentes grupos de estudantes. Ou seja, as especificidades de uma análise educacional impõem que o relevante em termos educacionais é a equivalência entre distribuições e não a igualdade absoluta do aprendizado.

A medida da desigualdade indica quais grupos sociais estão mais perto ou mais distante da situação de referência. Outras abordagens têm sido usadas para estudar desigualdades educacionais, como aquelas relatadas por Alves, Soares, Xavier (2016); Oliveira et al. (2013); Rodrigues, Rios-Neto, Pinto (2011); Soares, Alves e Xavier (2016); e Thomas, Wang e Fan (2001).

Para tratar desse assunto, este artigo está organizado da seguinte maneira: a segunda seção apresenta a metodologia de definição da distribuição de referência; nessa e nas outras seções usam-se os dados da Prova Brasil de 2005 a 2013 para concretizar a discussão. Na terceira seção, define-se a forma de cálculo da distância entre a distribuição de referência e a distribuição na qual nos interessa analisar a desigualdade e o indicador resultante. Na quarta seção, com esse referencial construído, analisam-se as diferenças associadas ao NSE, ao sexo, à cor-raça e à região. Finalmente, na última seção, destinada à discussão, coloca-se a proposta deste artigo no âmbito da literatura da área.

\section{DADOS E MEDIDAS}

Desde 1995, o Saeb implantou um sólido processo para medir o aprendizado dos alunos do quinto e nono anos do ensino fundamental em Leitura e Matemática. Criaram-se matrizes de especificação para as competências leitoras e 
matemáticas. Naturalmente, a medida de aprendizado é tão boa quanto sua matriz de especificação e os itens utilizados nos testes. Esse é um tema que tem recebido pouca atenção. Alertas importantes sobre as limitações da matriz do Saeb foram colocados por Ribeiro e Coscarelli (2010). As respostas dos alunos aos itens dos testes usados no Saeb são transformadas em escores pelo modelo de três parâmetros da Teoria de Resposta ao Item (KLEIN; FONTANIVE; MOURA, 2003). Esses escores recebem o nome técnico de proficiências. Com a inclusão de itens comuns nos testes aplicados aos estudantes das duas etapas de ensino incluídas na Prova Brasil e nos testes de dois ciclos contíguos, garante-se também uma escala única de medida que assim pode ser usada para monitoramento dos resultados ao longo dos anos. Neste artigo, utilizamos apenas as proficiências dos alunos das escolas estaduais e municipais que participaram da Prova Brasil, feita bianualmente, de 2005 a 2013.

\section{DISTRIBUIÇÃO DAS PROFICIÊNCIAS OBSERVADAS}

Conhecidas as proficiências dos alunos que se submeteram aos testes da Prova Brasil, pode-se estimar a densidade da distribuição dessas proficiências. ${ }^{3}$

O Gráfico 1 mostra as densidades estimadas das proficiências em Matemática do quinto e nono anos escolares para 2005 e 2013. A distribuição das proficiências do quinto ano de 2013 é claramente mais concentrada em valores mais altos da proficiência que a distribuição de 2005, refletindo a melhoria observada no período. No entanto, esse comportamento não é observado para o nono ano, etapa escolar em que a mudança constatada é de outra natureza.
3 Este artigo usa o método Kernel, metodologia sintetizada em Silverman (1986) e disponível em vários softwares, em particular no R ( $R$ DEVELOPMENT CORE TEAM, 2013) 


DISTRIBUIÇÕES DE REFERÊNCIA PARA PROFICIÊNCIAS

O objetivo desta seção é estabelecer as distribuições de referência para as proficiências medidas na Prova Brasil, isto é, a distribuição que deveria ser observada caso o aprendizado dos alunos estivesse na situação tomada como ideal. Apenas após a especificação dessas distribuições e da construção de uma interpretação pedagógica de seus diferentes valores é possível fazer julgamentos de valor sobre o desempenho observado dos alunos e, consequentemente, propor políticas públicas para o enfrentamento dos problemas encontrados.

Surpreendentemente, essa importante decisão ainda não alcançou um teor conclusivo no âmbito do Saeb e da Prova Brasil. O relatório do Saeb de 1997 lista, sem nenhuma justificativa, alguns valores desejados (os quais deixaram de ser referenciados nos relatórios técnicos dos Saebs posteriores). Nos últimos anos, algumas iniciativas fixaram valores de referência para os resultados de aprendizado. Primeiramente, a organização não governamental Todos pela Educação, antes de adotar as metas de desempenho para o sistema de educação básica que iria acompanhar, contratou estudos para sua definição. A introdução do Ideb pelo governo federal também exigiu a criação de metas de desempenho que, por sua vez, exigiu definição de valores a serem atingidos. As equipes técnicas envolvidas nas duas iniciativas tinham membros em comum e assim não surpreende a similaridade das soluções propostas. Em ambos os casos, usou-se a experiência do Programm for International Student Assessment (Pisa) como ponto de partida. Para detalhes, ver Brasil (2007) e Todos pela Educação (2008).

Em esforço similar, Soares (2009), no âmbito da definição do Índice de Desenvolvimento da Educação de São Paulo, também usando a referência do Pisa, propôs a utilização de quatro níveis para dar sentido normativo ao aprendizado dos alunos. Esses níveis foram usados em várias iniciativas, em particular na construção do Portal QEdu (2011), e serão usados também neste texto. As metas do Ideb, implicitamente, e do Todos pela Educação, explicitamente, consideram que a situação ideal é aquela em que 70\% dos estudantes têm proficiência nos níveis adequado ou avançado. A fixação de 
pontos de corte que definam níveis de aprendizado deveria ser feita por meio da interpretação pedagógica dos escores, conforme descrito, por exemplo, por Cizek e Bunch (2007). Essa pesquisa ainda não foi feita de forma completa no Brasil, ainda que grande avanço tenha sido alcançado com a introdução da plataforma Devolutivas pelo Inep, em 2015 (BRASIL, 2015).

Neste texto, usa-se para construção da distribuição de referência metodologia muito similar àquela empregada por Soares, Marotta e Delgado (2010). Para isso criou-se um "país típico" constituído por: Alemanha, Austrália, Áustria, Bélgica, Canadá, Coreia, Dinamarca, Espanha, Estados Unidos, Finlândia, França, Holanda, Inglaterra, Irlanda, Islândia, Itália, Japão, Noruega, Nova Zelândia, Portugal, Suécia e Suíça.

Para cada um desses países, usando-se o peso amostral de cada estudante, calcularam-se os valores dos 100 percentis da distribuição das proficiências no Pisa. Para Leitura, foram usados os dados de 2000 e para Matemática os de 2003, ciclos em que as respectivas escalas foram definidas. Cada percentil da distribuição do país típico foi definido como a média aritmética simples dos percentis de cada um dos países selecionados. Esse valor é denotado por $Z_{r}$, em que $r$ assume valores inteiros entre 0 e 100 e indica o percentil da distribuição. Paralelamente, os 100 percentis da distribuição de proficiência dos alunos brasileiros em Leitura e Matemática nos mesmos ciclos do Pisa também foram obtidos e denotados por $Y_{r}$.

Como os estudantes do país típico têm desempenhos maiores que os estudantes brasileiros, há diferença positiva entre os percentis desse país e os respectivos percentis dos estudantes brasileiros no mesmo teste do Pisa. Essa distância $\delta_{r}$, expressa em unidades do desvio padrão $-\sigma-$ da distribuição das proficiências dos alunos do Brasil no Pisa é dada por:

$$
\delta_{r}=\frac{Z_{r}-Y_{r}}{\sigma}
$$

O valor $\delta_{r}$ indica quantos desvios padrão cada percentil da distribuição dos alunos brasileiros no Pisa deveria aumentar 
para que os percentis da distribuição de desempenho dos estudantes brasileiros fossem iguais aos percentis do país típico. A Tabela 1 sintetiza e ilustra os cálculos feitos. O valor do desvio padrão empregado é igual a 95,6 obtido pelos dados ponderados do Brasil no Pisa Matemática 2003.

TABELA 1 - Diferenças entre o desempenho dos alunos brasileiros e alunos do país típico no Pisa 2003 em Matemática

\begin{tabular}{ccccc}
\hline $\begin{array}{c}\text { ORDEM DO } \\
\text { PERCENTIL }\end{array}$ & $\begin{array}{c}\text { VALOR DO } \\
\text { PERCENTIL } \\
\text { DOS ALUNOS } \\
\text { BRASILEIROS }\end{array}$ & $\begin{array}{c}\text { VALOR DO } \\
\text { PERCENTIL } \\
\text { DOS ALUNOS } \\
\text { DO PAÍS } \\
\text { TÍPICO }\end{array}$ & $\begin{array}{c}\text { DIFERENCA } \\
\text { EM PONTOS } \\
\text { ENTRE OS } \\
\text { PERCENTIS }\end{array}$ & $\begin{array}{c}\text { DIFERENÇA } \\
\text { EM DESVIOS } \\
\text { PADRÃO } \\
\text { ENTRE OS } \\
\text { PERCENTIS }\end{array}$ \\
\hline 5 & 221 & 367 & 146 & 1,53 \\
\hline 15 & 263 & 419 & 156 & 1,63 \\
\hline 30 & 302 & 466 & 164 & 1,71 \\
\hline 50 & 350 & 516 & 166 & 1,74 \\
\hline 75 & 419 & 579 & 159 & 1,66 \\
\hline 90 & 489 & 632 & 143 & 1,50 \\
\hline 95 & 539 & 663 & 124 & 1,30 \\
\hline
\end{tabular}

Fonte: Elaboração dos autores.

Para obter os percentis da distribuição de referência na escala Saeb aplicou-se a cada percentil da distribuição do Saeb de 1997 (ano de definição da escala) a translação por $\delta_{r}$, cuja construção foi detalhada anteriormente. Os percentis da distribuição de referência para o Saeb $X_{r}^{\prime}$ são obtidos pela seguinte expressão:

$$
X_{r}=X_{r}^{\prime}+\delta_{r} \cdot s
$$

Nessa expressão, $X_{r}$ é o valor na escala Saeb do percentil $r$ da distribuição de referência e $X_{r}^{\prime}$ é o mesmo percentil antes da translação, $s$ é o desvio padrão da distribuição do Saeb de 1997, que em 1997 foi de 44 pontos na escala Saeb para Matemática. A Tabela 2 sintetiza e ilustra os cálculos. 
TABELA 2 - Construção da distribuição de referência na métrica do Saeb para os alunos da oitava série - Matemática - do ensino fundamental

\begin{tabular}{cccc}
\hline PERCENTIL & $\begin{array}{c}\text { VALOR DOS } \\
\text { PERCENTIS DA } \\
\text { DISTRIBUIÇÃO } \\
\text { SAEB - 1997 }\end{array}$ & $\begin{array}{c}\text { TRANSLAÇÃO } \\
\text { NECESSÁRIA EM } \\
\text { DESVIOS PADRÃO }\end{array}$ & $\begin{array}{c}\text { PERCENTIS DA } \\
\text { DISTRIBUIÇÃO DE } \\
\text { REFERÊNCIA }\end{array}$ \\
\hline 05 & 170 & 1,53 & 237 \\
\hline 15 & 192 & 1,63 & 264 \\
\hline 30 & 223 & 1,71 & 318 \\
\hline 50 & 242 & 1,74 & 351 \\
\hline 75 & 278 & 1,66 & 382 \\
\hline 90 & 316 & 1,50 & 398 \\
\hline 95 & 341 & 1,30 & \\
\hline
\end{tabular}

Fonte: Elaboração dos autores.

Conhecidos os 100 percentis $r$ da distribuição de refe-

4 Para isso, usou-se o fato de que se $F$ é a função de densidade acumulada da distribuição $X$, a transformação $F(X)$ tem distribuição uniforme $F(X) \sim U$. Essa transformação é amplamente usada na simulação de distribuições estatísticas e está bem descrita, por exemplo, em Angus (1994) rência do Saeb, a amostra dessa distribuição foi gerada. ${ }^{4} \mathrm{~A}$ Tabela 3 descreve as distribuições obtidas e mostra que o percentil 30 da distribuição de referência é próximo da meta escolhida pela organização não governamental Todos pela Educação.

TABELA 3 - Valores dos parâmetros das distribuições de referência

\begin{tabular}{lcccc}
\hline COMPETÊNCIA & $\begin{array}{c}\text { MÉDIA } \\
(\mu)\end{array}$ & $\begin{array}{c}\text { DESVIO } \\
\text { PADRÃO }(\sigma)\end{array}$ & 30 PERCENTIL & $\begin{array}{c}\text { METAS } \\
\text { TODOS PELA } \\
\text { EDUCAÇÃO }\end{array}$ \\
\hline LEITURA: & & & & \\
Quinto Ano & 240,7 & 49,2 & 213,5 & 200 \\
Nono Ano & 304,6 & 54,9 & 278,3 & 275 \\
\hline MATEMÁTICA: & & & & 225 \\
Quinto Ano & 261,5 & 41,3 & 239,2 & 300 \\
Nono Ano & 321,2 & 48,4 & 295,0 & \\
\hline
\end{tabular}

Fonte: Elaboração dos autores.

O Gráfico 2 mostra as distribuições de referência escolhidas para as duas disciplinas e dois anos escolares avaliados pela Prova Brasil com as respectivas distribuições referentes a 2005 e 2013. 


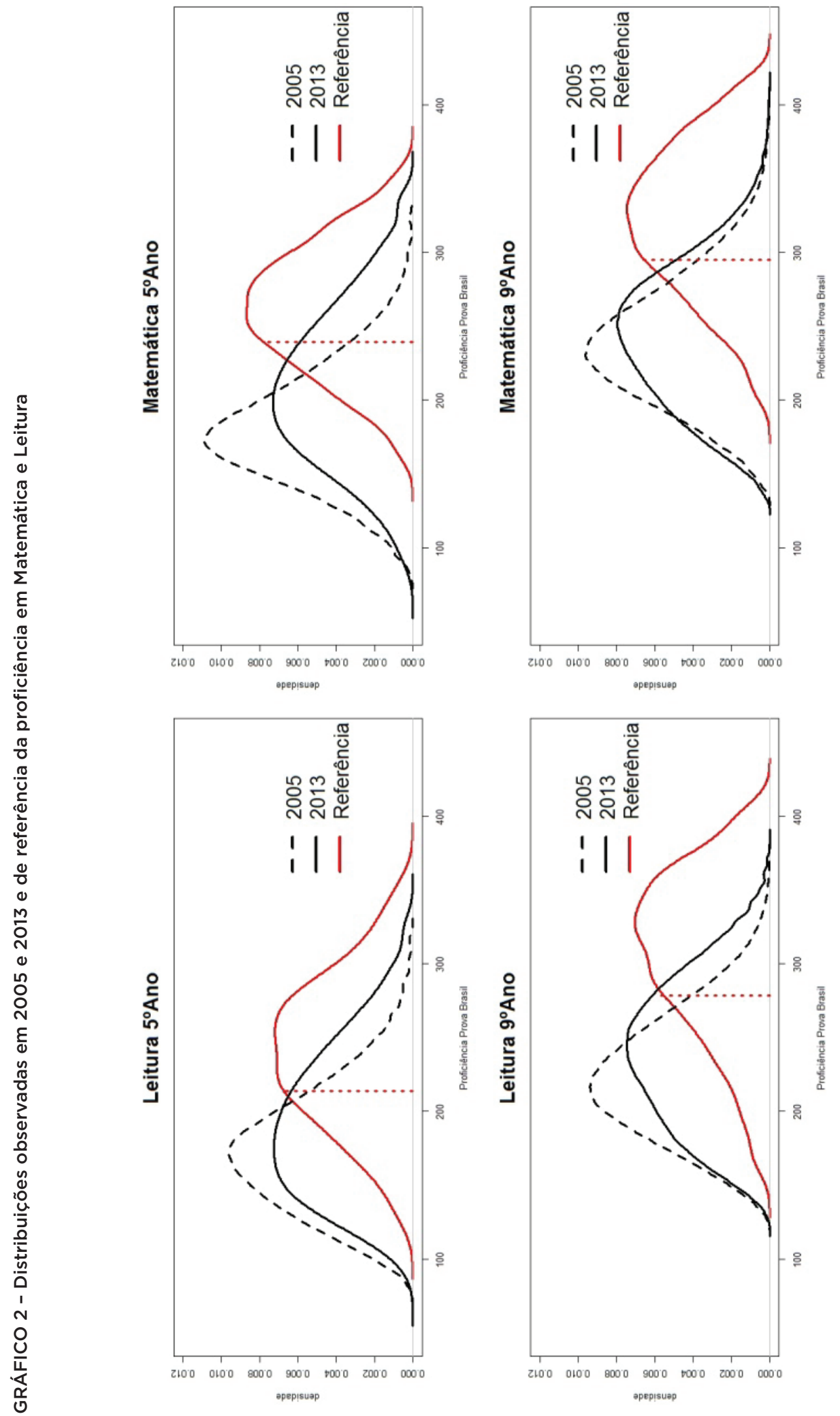


As matrizes de referência do Saeb e do Pisa são diferentes e, portanto, os conhecimentos e habilidades medidos nos dois testes também são. Desse modo, mesmo que a distribuição de referência construída com os dados do Saeb fosse atingida, não há garantia de que o desempenho dos alunos brasileiros no Pisa seria equivalente ao desempenho dos alunos do país típico.

Na educação, embora não faça sentido considerar igualdade de desempenho entre estudantes como um ideal, pode-se definir também situações extremas, úteis para a interpretação dos valores das distâncias. Por isso, neste texto, considerou-se também a situação extrema em que todos os estudantes têm proficiência no nível avançado. Essa situação extrema, mas não impossível, cria limites naturais para as distribuições de proficiência dos vários grupos de estudantes que fazem os testes do Saeb.

\section{INDICADORES DE QUALIDADE}

\section{E DESIGUALDADE EDUCACIONAL}

A distância entre a distribuição observada e a distribuição de referência é tomada neste texto como medida de qualidade da educação, já que mede quão longe da situação ideal a situação real está. Nesse referencial, a desigualdade educacional é estudada calculando-se as distâncias das distribuições de desempenho relativas a diferentes grupos de estudantes até a distribuição de referência e comparando-se os valores obtidos, exatamente como é feito nos estudos de desigualdade de renda.

Kullback e Leibler (1951) introduziram uma forma muito eficaz de medir a distância entre duas distribuições estatísticas, doravante denominada KL. Essa medida capta diferenças de qualquer natureza entre as duas distribuições. Por exemplo, no debate educacional, é importante considerar tanto a distância entre os estudantes de melhor desempenho até os valores altos da referência, como a distância dos estudantes de pior desempenho até os valores baixos da distribuição de referência. Ambas as situações caracterizam problemas educacionais que devem ser evidenciados pela medida de qualidade. No entanto, a completa compreensão da medida KL 
exige sofisticados conhecimentos matemáticos. Handcock e Morris (2006), utilizando noção de distribuição relativa, apresentada a seguir de forma simplificada, facilitaram o uso dessa medida nas ciências sociais. Esses autores usam um gráfico para explicar o formalismo matemático da medida.

O Gráfico 3 apresenta as distribuições das proficiências dos estudantes do quinto ano na Prova Brasil em Leitura para 2005 e 2013 com a respectiva distribuição de referência, que está representada por uma reta, enquanto as distribuições dos dois ciclos da Prova Brasil se transformam em curvas que aparecem abaixo da reta. O gráfico ilustra, por meio de áreas, a distância existente entre a situação real e a distribuição de referência. No entanto, essas áreas são equivalentes à diferença entre as médias das distribuições. Como é amplamente conhecido, as médias registram um aspecto muito específico das distribuições que, entretanto, pode ser dominante em alguns casos. A medida KL, por outro lado, é capaz de captar qualquer tipo de diferença, o que justifica seu uso apesar de seu hermetismo matemático.

GRÁFICO 3 - Representação da densidade acumulada de $g(r)$ para as proficiências em Leitura dos estudantes de quinto ano em 2005 e 2013

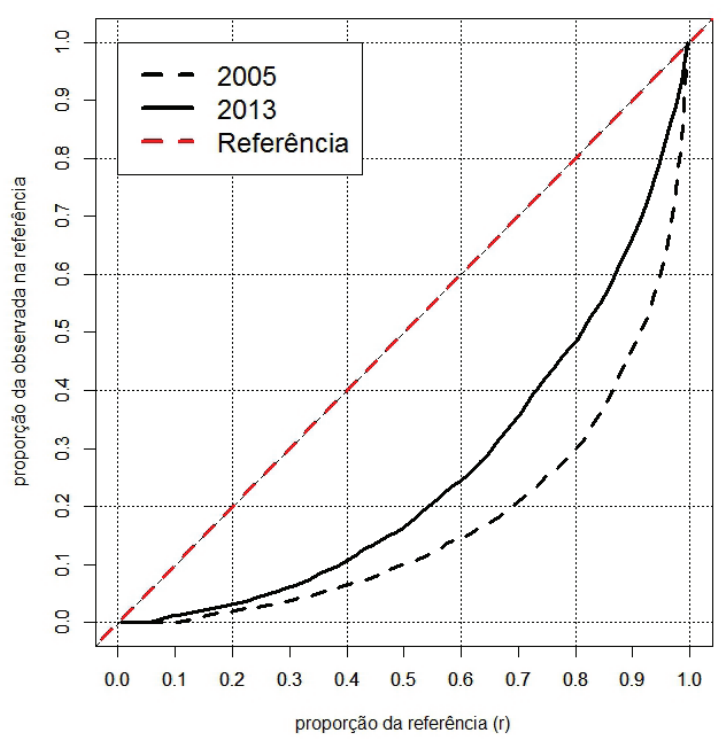


Soares (2006) e depois Soares, Marotta e Delgado (2010) usaram as áreas entre as curvas e a reta como medida de distância entre as distribuições.

De forma sucinta em notação matemática, a distribuição KL pode ser introduzida da seguinte maneira: denotando por $f_{1}$ e $f_{2}$ as densidades das distribuições de referência e observada, respectivamente, a distância KL é definida por:

$$
K L\left(f_{1} ; f_{2}\right)=\int_{-\infty}^{\infty} f_{1}(X) \cdot \log \left(\frac{f_{1}(X)}{f_{2}(X)}\right) d X
$$

Shlens (2014) explica a origem dessa expressão, pouco intuitiva, mostrando que ela é, na realidade, uma aproximação para a função de verossimilhança. No caso de as distribuições $f_{1}$ e $f_{2}$ serem discretas, a distância KL é uma aproximação para a verossimilhança de se observar $f_{2}$ quando na realidade o modelo gerador dos dados é $f_{1}$.

O uso dessa distância tornou-se mais comum nas ciências sociais a partir dos trabalhos de Handcock e Morris (1998 e 2006). Primeiramente esses autores mostraram a utilidade de se introduzir o conceito de densidade relativa definida por:

$$
g(r)=\frac{f_{\mathrm{Ref}}\left(X_{r}\right)}{f_{\mathrm{Obs}}\left(X_{r}\right)}
$$

onde $f_{\text {Ref }}$ é a densidade da distribuição de referência, $f_{\text {obs }}$ é a densidade da distribuição observada, o $r$ é qualquer quantil da distribuição $r=F(X)$, tal que $0 \leq r \leq 1$. Com essa notação, a distância KL é definida como:

$$
K L\left(f_{\text {Ref }} ; f_{\text {Obs }}\right)=\int_{0}^{1} \log (g(r)) \cdot g(r) d r
$$

A utilidade da densidade relativa é mais facilmente entendida se sua densidade acumulada é considerada. No Gráfico 3, o eixo horizontal recebe os quantis da distribuição de referência $(r)$ e, no eixo vertical, os quantis da distribuição observada na escala da distribuição de referência. Naturalmente, quando, no eixo horizontal, são colocados os quantis 
da distribuição de referência, obtém-se uma reta de inclinação igual a $45^{\circ}$, já que, nessa situação, as distribuições comparadas são iguais.

Quando no, eixo horizontal, está a distribuição de uma situação observada, i.e., as proficiências dos estudantes de uma escola ou de um sistema de ensino, obtém-se uma curva que fica abaixo da reta. Quanto menor a área entre a reta e a curva associada a um dado grupo, mais próximo se está da distribuição de referência. Embora essa figura seja muito similar às curvas de Lorenz, na realidade a análise que leva ao coeficiente de Gini é apenas um caso particular dessa formulação, como explicado por Handcock e Morris (2006).

\section{INTERPRETAÇÃO DA MEDIDA DE DISTÂNCIA}

O objetivo desta seção é mostrar quais valores da medida KL são relevantes em termos educacionais. Para isso, utilizam-se dois resultados sobre a escala do Saeb. O primeiro refere-se aos níveis normativos. Soares (2006) tomou valores próximos aos percentis 5,30 e 75 da distribuição de referência, como os pontos de corte para níveis que receberam denominação de abaixo do básico, básico, adequado e avançado. Nesta seção, vamos usar, para comparação com a distribuição observada, além da distribuição de referência, a distribuição em que todos os estudantes têm proficiências no nível avançado. ${ }^{5}$ Embora seja possível, isso é pouco frequente.

A Tabela 3 mostra que a diferença entre os valores fixados para o limite inferior do nível adequado no quinto e nono anos é de 75 pontos. Esse valor indica, portanto, o número de pontos na escala do Saeb que cada estudante deveria acumular em quatro anos de escolarização. Assim sendo, é razoável tomar 20 pontos como o aumento esperado por ano de escolarização.

A Tabela 4 fornece os valores da distância KL entre as distribuições de proficiência observadas e as respectivas distribuições de referência, bem como em relação a uma distribuição extrema em que todos os estudantes estão no nível avançado.
5 Para receber o código em $\mathrm{R}$ que permite obter o valor da medida entre duas distribuições quaisquer, contatar os autores por e-mail. 
TABELA 4 - Valores da distância KL entre as distribuições de proficiência observadas e as respectivas distribuições de referência e extrema, de cada ano e disciplina

\begin{tabular}{lcc}
\hline ANO & $K L_{\text {REFERENCIA }}$ & $K L_{\text {AVANCADO }}$ \\
\hline LEITURA 5 ANO: & 0,95 & 3,38 \\
2005 & 0,42 & 2,54 \\
2013 & & \\
\hline MATEMÁTICA 5 ANO: & 1,50 & 4,00 \\
2005 & 0,57 & 2,44 \\
2013 & & \\
\hline LEITURA 9ㅇ ANO: & 1,15 & 4,24 \\
2005 & 0,72 & 3,51 \\
2013 & & 4,31 \\
\hline MATEMÁTICA 9 ANO: & 1,26 & 4,11 \\
2005 & 1,09 & \\
2013 & & \\
\hline
\end{tabular}

Fonte: Elaboração dos autores.

A comparação dos valores das linhas referentes a 2005 e 2013 mostra que houve melhoria no desempenho dos estudantes brasileiros, já que diminuíram as distâncias registradas na Tabela 4 entre a distribuição observada e a distribuição de referência para uma mesma disciplina e ano escolar. Esse é um resultado bastante conhecido, pois aparece independentemente da métrica usada para sintetizar as distribuições de desempenho em cada ano.

A Tabela 5 apresenta valores da distância KL entre distribuições, cujo sentido normativo é conhecido. A apreciação desses valores é fundamental para atribuir sentido pedagógico à medida KL. 
TABELA 5 - Porcentagem de estudantes nos níveis da Prova Brasil, Leitura quinto ano para 2005 e 2013, e distribuição de referência e translações de 2013

\begin{tabular}{cccccc}
\hline ANO & $\begin{array}{c}\text { ABAIXO DO } \\
\text { BÁSICO }\end{array}$ & BÁSICO & ADEQUADO & AVANÇADO & KL $_{\text {REF }}$ \\
\hline \multicolumn{7}{c}{2005} & $29,7 \%$ & $44,6 \%$ & $21,3 \%$ & $4,4 \%$ & 0,953 \\
\hline 2013 & $21,6 \%$ & $35,9 \%$ & $28,5 \%$ & $14,0 \%$ & 0,423 \\
\hline Referência & $3,3 \%$ & $18,4 \%$ & $34,6 \%$ & $43,8 \%$ & 0 \\
\hline PROVA BRASIL 2013 COM TRANSLAÇÕES: & $35,5 \%$ & $31,1 \%$ & $18,4 \%$ & 0,274 \\
\hline $2013+10$ & $15,0 \%$ & $33,9 \%$ & $33,2 \%$ & $23,6 \%$ & 0,157 \\
\hline $2013+20$ & $9,3 \%$ & $30,9 \%$ & $34,7 \%$ & $29,4 \%$ & 0,078 \\
\hline $2013+30$ & $5,0 \%$ & & & \\
\hline
\end{tabular}

Fonte: Elaboração dos autores.

Nas duas primeiras linhas da Tabela 5, estão as distribuições de Leitura no quinto ano para os ciclos de 2005 e 2013 da Prova Brasil. Esses são os melhores resultados do ensino fundamental brasileiro. Como observado, houve melhoria substancial entre 2005 e 2013, evidenciada nessa tabela pelo aumento do percentual de estudantes no nível avançado e na diminuição no nível abaixo do básico. Como se constata na última coluna da Tabela 5, a melhoria entre 2005 e 2013 equivale a uma diminuição de 0,53 no valor da medida KL. Portanto esse é um valor da medida KL pedagogicamente significativo.

No entanto, os resultados tanto de 2005 como de 2013 são ainda muito ruins, pois o percentual de alunos nos dois níveis mais altos em 2013 é muito menor que 70\%, uma meta a ser atingida. Transformar a distribuição de 2013 na distribuição de referência equivale a reduzir a medida KL em 0,42.

As três linhas seguintes fornecem o valor da medida KL e dos percentuais para distribuições obtidas a partir da distribuição de 2013 com translações sucessivas de 10, 20 e 30 pontos. Como se observa, comparando-se a medida KL para as três distribuições obtidas por uma translação de 10 pontos, esse valor induz na medida KL uma mudança aproximada de 0,10 .

Tomando todas essas evidências conjuntamente, adotamos os seguintes pontos de corte para interpretar a distância KL: 
QUADRO 1 - Pontos de corte interpretativos da medida KL

\begin{tabular}{|ccccc|}
\hline \multicolumn{5}{|c|}{ Valor normativo das distâncias KL } \\
\hline Pequena & Baixa & Alta & Muito Alta & Enorme \\
\hline$[0,0 ; 0,10]$ & {$[0,10 ; 0,30]$} & {$[0,30 ; 0,50]$} & {$[0,50 ; 1,0]$} & $>1,0$ \\
\hline
\end{tabular}

Fonte: Elaboração dos autores.

Com esses referenciais, pode-se observar que a maioria das comparações neste artigo se dá entre distribuições de proficiência observadas que estão pedagogicamente muito distantes das respectivas distribuições de referência. Isso é fruto do fato de que o aprendizado de Leitura e Matemática dos estudantes de ensino fundamental brasileiro é ainda muito aquém dos patamares internacionais e, portanto, a respectiva distribuição está longe da distribuição de referência.

\section{DESIGUALDADES DE APRENDIZADO}

Nesta seção, apresentamos a distância KL para grupos definidos por sexo, raça-cor, NSE e região do país e agrupados pelo ano-escolar. Para obter a estimativa do decréscimo médio observado no período, adotamos a média aritmética dos quatro decréscimos obtidos pela comparação dos ciclos de 2005 com 2007, 2007 com 2009, 2009 com 2011 e 2011 com 2013. Esse decréscimo médio pode ser transformado em número de anos necessários até que a distribuição da proficiência de cada grupo fique igual à distribuição de referência. Esse valor é apresentado na última coluna das tabelas 7 a 10.

\section{QUINTO ANO}

\section{SEXO}

A Tabela 6 mostra os valores da distância KL entre os estudantes do sexo masculino e feminino. 
TABELA 6 - Valores da distância KL até a distribuição de referência dos grupos definidos por sexo e ano da Prova Brasil - quinto ano

\begin{tabular}{c|c|c|c|c|c|c}
\hline SEXO/DISCIPLINA & $\mathbf{2 0 0 5}$ & $\mathbf{2 0 0 7}$ & $\mathbf{2 0 0 9}$ & $\mathbf{2 0 1 1}$ & $\mathbf{2 0 1 3}$ & $\begin{array}{c}\text { ANOS ATÉ } \\
\text { REFERÊNCIA }\end{array}$ \\
\hline Leitura - Masculino & 1,06 & 1,06 & 0,81 & 0,68 & 0,52 & 30 \\
\hline Leitura - Feminino & 0,83 & 0,84 & 0,57 & 0,42 & 0,31 & 19 \\
\hline Matemática- Masculino & 1,43 & 1,03 & 0,70 & 0,59 & 0,52 & 18 \\
\hline Matemática - Feminino & 1,53 & 1,15 & 0,79 & 0,69 & 0,57 & 18 \\
\hline
\end{tabular}

Fonte: Elaboração dos autores.

Primeiramente deve-se observar o decréscimo substancial na distância KL ocorrido entre 2005 e 2013 entre a distribuição observada e a distribuição de referência. Ocorreram melhorias tanto em Leitura como em Matemática, ainda que mais rápidas em Matemática, tanto para os meninos como para as meninas. Entretanto, as diferenças entre as distâncias das distribuições referentes aos dois sexos praticamente não mudam. Em Leitura, essa distância estava em 0,23 em 2005 e em 0,22 em 2013. A diferença entre as distâncias das distribuições referentes aos dois sexos até a distribuição de referência é menor em Matemática que em Leitura. Ou seja, em termos substantivos, no quinto ano, as meninas estão perto dos meninos em Matemática e os superam amplamente em Leitura. No entanto, serão necessários mais de 20 anos até que o desempenho dos grupos definidos por sexo chegue aos níveis desejados.

\section{RAÇA-COR}

TABELA 7 - Valores da distância KL até a distribuição de referência dos grupos definidos por cor-raça e ano da Prova Brasil - quinto ano

\begin{tabular}{c|c|c|c|c|c|c}
\hline $\begin{array}{c}\text { COR-RAÇA/ } \\
\text { DISCIPLINA }\end{array}$ & $\mathbf{2 0 0 5}$ & $\mathbf{2 0 0 7}$ & $\mathbf{2 0 0 9}$ & $\mathbf{2 0 1 1}$ & $\mathbf{2 0 1 3}$ & $\begin{array}{c}\text { ANOS ATÉ } \\
\text { REFERÊNCIA }\end{array}$ \\
\hline Leitura - Branco & 0,76 & 0,80 & 0,55 & 0,38 & 0,27 & 17 \\
\hline Leitura - Pardo & 0,99 & 0,97 & 0,70 & 0,57 & 0,40 & 21 \\
\hline Leitura - Preto & 1,35 & 1,30 & 1,08 & 0,85 & 0,69 & 33 \\
\hline Matemática - Branco & 1,26 & 0,90 & 0,59 & 0,46 & 0,39 & 14 \\
\hline Matemática - Pardo & 1,55 & 1,13 & 0,77 & 0,68 & 0,54 & 17 \\
\hline Matemática - Preto & 1,98 & 1,59 & 1,20 & 1,03 & 0,90 & 26 \\
\hline
\end{tabular}

Fonte: Elaboração dos autores.

A situação das desigualdades em relação à raça-cor mostra que houve melhoria substancial de todos os grupos entre 
2005 e 2013. No entanto, a distância de todos esses grupos até a distribuição de referência é grande. O tempo necessário para que o grupo dos que se autodeclaram pretos atinja a situação ideal é duas vezes maior que o tempo necessário para os que se autodeclaram brancos. Isso mostra que, com intervenções para a melhoria do desempenho, é necessário pensar formas de fazer com que os grupos de alunos pardos e pretos atinjam esse objetivo rapidamente. O movimento entre as distribuições dos grupos definidos por raça-cor é maior que aquele observado nos grupos definidos por sexo. Esse fato se reflete no número de anos necessários para se chegar à distribuição de referência.

\section{NÍVEL SOCIOECONÔMICO}

TABELA 8 - Valores da distância KL até a distribuição de referência dos grupos definidos por nível socioeconômico (NSE) e ano da Prova Brasil - quinto ano

\begin{tabular}{c|c|c|c|c|c|c}
\hline COR-RAÇA/DISCIPLINA & $\mathbf{2 0 0 5}$ & $\mathbf{2 0 0 7}$ & $\mathbf{2 0 0 9}$ & $\mathbf{2 0 1 1}$ & $\mathbf{2 0 1 3}$ & $\begin{array}{c}\text { ANOS ATÉ } \\
\text { REFERÊNCIA }\end{array}$ \\
\hline Leitura - Baixo - NSE & 1,11 & 1,19 & 1,11 & 0,97 & 0,91 & 145 \\
\hline Leitura - Médio -NSE & 0,90 & 0,96 & 0,71 & 0,60 & 0,50 & 40 \\
\hline Leitura - Alto- NSE & 0,64 & 0,68 & 0,43 & 0,33 & 0,23 & 17 \\
\hline Matemática - Baixo - NSE & 1,71 & 1,44 & 1,24 & 1,17 & 1,12 & 60 \\
\hline Matemática - Médio -NSE & 1,43 & 1,11 & 0,77 & 0,72 & 0,67 & 28 \\
\hline Matemática - Alto - NSE & 1,10 & 0,77 & 0,46 & 0,38 & 0,33 & 13 \\
\hline
\end{tabular}

Fonte: Elaboração dos autores.

A Tabela 8 mostra a dramática situação das desigualdades educacionais quando os alunos são separados por grupos socioeconômicos. O indicador de NSE foi calculado usando-se a metodologia definida por Alves, Soares e Xavier (2014). As diferenças observadas são muito grandes. Os alunos de NSE baixo precisam de até oito vezes mais tempo para chegar à distribuição de referência que seus colegas de NSE alto. Mas para esses estudantes o tempo estimado é tão grande, tanto em Leitura como em Matemática - 145 e 60 anos - que isso nunca ocorrerá. Ou seja, o sistema educacional brasileiro deve encontrar outras formas de atender estudantes de NSE mais baixo que trazem poucos conhecimentos escolares de casa e, portanto, dependem completamente da escola para adquirir os conhecimentos e habilidades que precisam 
para a vida. Se não houver mudanças no sistema, esses alunos continuarão a ser excluídos do sistema pelo seu baixo aprendizado.

Ao se analisar a distância KL dos grupos ao longo dos ciclos, nota-se ainda que a distância entre o grupo de NSE mais alto e o mais baixo está crescendo, com algumas flutuações. Isso mostra que a melhoria que ocorreu nos últimos anos beneficiou basicamente os alunos de NSE mais alto, fato já mostrado por Alves, Soares e Xavier (2016).

\section{REGIÃO}

TABELA 9 - Valores da distância KL até a distribuição de referência dos grupos definidos por Região e ano da Prova Brasil - quinto ano

\begin{tabular}{c|c|c|c|c|c|c}
\hline REGIÃO/DISCIPLINA & $\mathbf{2 0 0 5}$ & $\mathbf{2 0 0 7}$ & $\mathbf{2 0 0 9}$ & $\mathbf{2 0 1 1}$ & $\mathbf{2 0 1 3}$ & $\begin{array}{c}\text { ANOS ATÉ } \\
\text { REFERERNCIA }\end{array}$ \\
\hline Leitura - Norte & 1,27 & 1,28 & 1,09 & 0,92 & 0,75 & 46 \\
\hline Leitura - Nordeste & 1,39 & 1,40 & 1,26 & 1,04 & 0,87 & 53 \\
\hline Leitura - Sudeste & 0,73 & 0,78 & 0,48 & 0,38 & 0,24 & 15 \\
\hline Leitura- Sul & 0,81 & 0,82 & 0,59 & 0,40 & 0,24 & 13 \\
\hline Leitura - Centro-Oeste & 0,98 & 0,94 & 0,64 & 0,48 & 0,36 & 18 \\
\hline Matemática - Norte & 2,06 & 1,67 & 1,35 & 1,20 & 1,02 & 31 \\
\hline Matemática - Nordeste & 2,11 & 1,73 & 1,51 & 1,31 & 1,14 & 37 \\
\hline Matemática - Sudeste & 1,24 & 0,88 & 0,49 & 0,42 & 0,34 & 12 \\
\hline Matemática - Sul & 1,25 & 0,87 & 0,59 & 0,42 & 0,32 & 11 \\
\hline Matemática - Centro-Oeste & 1,53 & 1,12 & 0,76 & 0,61 & 0,52 & 16 \\
\hline
\end{tabular}

Fonte: Elaboração dos autores.

A análise das desigualdades por região mostra quadro muito similar ao verificado em relação às outras variáveis. As informações dessa tabela mostram que nas regiões Sul e Sudeste é possível que se atinjam as metas, sejam as do Ideb, sejam as do Todos pela Educação, mas nas outras regiões isso não ocorrerá, pois o tempo necessário é demasiadamente grande. Deve-se ainda observar que as desigualdades entre as regiões não estão diminuindo ao longo dos ciclos. O país continua e continuará profundamente dividido, também na educação, se não houver intervenções mais efetivas nessa área.

\section{CRITÉRIOS MÚLTIPLOS}

As características sociodemográficas consideradas individualmente nas seções anteriores, na realidade, influenciam 
o aprendizado do estudante conjuntamente. Assim, para se produzir uma descrição mais completa das desigualdades educacionais, é preciso considerar também grupos criados por fatores múltiplos. A Tabela 10 mostra os resultados da distância KL para os grupos de meninas, pretas e de NSE baixo e meninos, brancos e de NSE alto.

TABELA 10 - Valores da distância KL até a distribuição de referência dos grupos 1 de Meninas, Pretas e de NSE Baixo e do grupo 2 de Meninos, Brancos e de NSE Alto

\begin{tabular}{c|c|c|c|c|c|c}
\hline GRUPO/DISCIPLINA & $\mathbf{2 0 0 5}$ & $\mathbf{2 0 0 7}$ & $\mathbf{2 0 0 9}$ & $\mathbf{2 0 1 1}$ & $\mathbf{2 0 1 3}$ & $\begin{array}{c}\text { ANOS ATÉ } \\
\text { REFERÊNCIA }\end{array}$ \\
\hline Grupo 1 - Leitura & 1.31 & 1.31 & 1.17 & 0.97 & 0.93 & 78 \\
\hline Grupo 2 - Leitura & 0.60 & 0.65 & 0.40 & 0.30 & 0.19 & 14 \\
\hline Grupo 1 - Matemática & 2.12 & 1.80 & 1.54 & 1.48 & 1.36 & 57 \\
\hline Grupo 2 - Matemática & 0.89 & 0.57 & 0.30 & 0.22 & 0.20 & 9 \\
\hline
\end{tabular}

Fonte: Elaboração dos autores.

Esses dois grupos representam estudantes que, embora nominalmente no mesmo ano escolar, do ponto de vista de aprendizado, estão em situações muito distintas. As estudantes do Grupo 1 nunca vão chegar a ter o aprendizado dos estudantes do Grupo 2 e as desigualdades entre os dois grupos também não desaparecerão se o sistema educacional brasileiro continuar a utilizar apenas as políticas atuais.

\section{NONO ANO}

Tabelas como as apresentadas na seção anterior foram construídas para o nono ano. Os resultados, socialmente devastadores, são fáceis de resumir. Nenhum grupo dos definidos pelos critérios considerados chegará à situação ideal, já que o número de anos estimado para isso ultrapassa, em todos os casos, os 50 anos. Como a qualidade, medida pela distância KL, é péssima para todos os grupos, a discussão de desigualdades perde proeminência. No entanto, mesmo na atual indigência dos aprendizados, as desigualdades encontradas para os grupos definidos por sexo, raça-cor, NSE e região e pelos critérios múltiplos usados para a construção da Tabela 10 são enormes. 


\section{DISCUSSÃO}

O estudo da desigualdade, em qualquer situação, exige que se descreva qual será o desvio em relação à situação de igualdade que será enfatizada. Ou seja, é preciso definir a situação de igualdade e a maneira de delimitar a distância até ela. Neste artigo argumentamos que a noção de igualdade em educação deve considerar grupos de estudantes, não indivíduos. Assim, a igualdade ocorre quando os grupos de estudantes, definidos por qualquer critério, têm a mesma distribuição. No entanto, interessa pouco ter a mesma distribuição se seus valores não correspondem a aprendizados em nível que habilitem o estudante à inserção produtiva e pessoalmente satisfatória na sociedade. Por isso, neste texto criou-se uma distribuição de referência utilizando-se interpretação normativa dos resultados obtida com a comparação internacional.

Qualquer indicador que sintetize o comportamento global de uma unidade de análise enseja uma hierarquização entre as unidades. Embora útil, por exemplo, para verificar o aumento ou diminuição do indicador e dar a ele uma primeira interpretação substantiva, a mera hierarquização é pouco informativa. Interessa saber se o nível do indicador é adequado ou não para as finalidades de monitoramento ou avaliação construídas. Ou ainda, se a diferença entre o indicador calculado para dois grupos ou em dois momentos do tempo é relevante substantivamente.

Essa formulação permite tratar os conceitos de qualidade e desigualdade em educação de uma mesma maneira. A qualidade é medida como a distância até a distribuição de referência, valor que mostra quão longe da situação de igualdade, definida como ideal, a situação real está. As desigualdades são analisadas comparando-se as medidas de distâncias das distribuições de diferentes grupos até a distribuição de referência. Essas mesmas comparações são feitas no estudo das desigualdades de renda, no qual a medida de distância até a situação ideal é o coeficiente de Gini.

Nos estudos de distribuição de renda, não se discute qual seria a renda adequada para os indivíduos de uma sociedade. Toda a análise se concentra na divisão igualitária da renda 
existente, mesmo que seja insuficiente para atender às necessidades das pessoas. No entanto, além da desigualdade, há o problema da pobreza. Na formulação usada neste texto, o equivalente educacional de pobreza foi incluído quando a distribuição de referência foi construída.

A qualidade da educação, nestes últimos 10 anos, foi monitorada no âmbito do governo federal pelo Ideb e no âmbito da sociedade civil pelo movimento Todos pela Educação. Ambos os processos de monitoramento não consideraram as desigualdades nos aprendizados, que, como mostradas neste texto, são muito grandes. A medida KL introduzida neste artigo é uma proposta concreta de indicador para o monitoramento das desigualdades entre grupos de estudantes. Em um país com histórico tão grande de desigualdades, esse monitoramento impõe-se como necessidade ética.

\section{AGRADECIMENTOS}

Os autores agradecem aos pareceristas, cujos comentários ajudaram a tornar o texto mais claro, e à Maria Teresa Gonzaga Alves, Izabel da Costa Fonseca e Elizabeth Leo, por leituras críticas e sugestões ao longo do desenvolvimento da pesquisa relatada neste artigo.

\section{REFERÊNCIAS}

ALVES, Maria Teresa Gonzaga; SOARES, José Francisco; XAVIER, Flavia Pereira. Índice socioeconômico das escolas de educação básica brasileiras. Ensaio: Avaliação e Políticas Públicas em Educação, Rio de Janeiro, v. 22, n. 84 , p. 671-703, set. 2014.

ALVES, Maria Teresa Gonzaga; SOARES, José Francisco; XAVIER, Flavia Pereira. Desigualdades educacionais no ensino fundamental de 2005 a 2013: hiato entre grupos sociais. Belo Horizonte. 2016.

ANGUS, John E. The probability integral transform and related results. Siam Review, v. 36, n. 4, p. 652-654, 1994.

BRASIL. Constituição (1988). Constituição da República Federativa do Brasil: Texto Constitucional promulgado em 5 de outubro de 1988, com as alterações anotadas pelas Emendas Constitucionais n ${ }^{\text {os }} 1 / 92$ a 44/2004 e pelas Emendas Constitucionais de Revisão n ${ }^{\text {os }} 1$ a 6/94. Brasília, DF: Senado Federal, Subsecretaria de Edições Técnicas, 2004. 438 p. 
BRASIL. Instituto Nacional de Estudos e Pesquisas Educacionais Anísio Teixeira. Nota técnica: Índice de Desenvolvimento da Educação Básica - Ideb. Metodologia da concepção do IDEB. Brasília, DF: Inep, 2007.

CIZEK, Gregory J.; BUNCH, Michael B. Standard setting: a guide to establishing and evaluating performance standards on tests. Thousand Oaks, CA: Sage, 2007.

HANDCOCK, Mark S.; MORRIS, Martina. Relative distribution methods. Sociological Methodology, v. 28, n. 1, p. 53-97, 1998.

HANDCOCK, Mark S.; MORRIS, Martina. Relative distribution methods in the social sciences. New York: Springer Science \& Business Media, 2006.

KLEIN, R.; FONTANIVE, N. S.; MOURA, F. Utilização da teoria de resposta ao item no Sistema Nacional de Avaliação da Educação Básica. Ensaio: Avaliação e Políticas Públicas em Educação, Rio de Janeiro, v. 11, n. 40, p. 283-296, jul./set. 2003.

KULLBACK, Solomon; LEIBLER, Richard A. On information and sufficiency. The Annals of Mathematical Statistics, v. 22, n. 1, p. 79-86, 1951.

OLIVEIRA, Romualdo Portela et al. Análise das desigualdades intraescolares no Brasil. 2013: projeto de pesquisa. São Paulo: Universidade de São Paulo, 2013. Disponível em: <http://www.fvc.org.br/estudos-e-pesquisas/2012/pdf/ relatorio-final-analise-desigualdades-intraescolares-brasil.pdf $>$. Acesso em: 19 set. 2016.

QEdu. Use dados. Transforme a educação. 2011. Disponível em: <http://www.qedu.org.br/>. Acesso em: 3 jul. 2016.

R DEVELOPMENT CORE TEAM. R: a language and environment for statistical computing. Viena, Austria, 2013. Disponível em: <R-project.org/>. Acesso em: 6 dez. 2016.

RIBEIRO, Ana Elisa; COSCARELLI, Carla Viana. O que dizem as matrizes de habilidades sobre a leitura em ambientes digitais. Educação em Revista, Belo Horizonte, v. 26, n. 3, p. 317, 2010.

RODRIGUES, Clarissa Guimarães; RIOS-NETO, Eduardo Luiz Gonçalves; PINTO, Cristine Campos de Xavier. Diferenças intertemporais na média e distribuição do desempenho escolar no Brasil: o papel do nível socioeconômico, 1997 a 2005. Revista Brasileira de Estudos Populares, Rio de Janeiro, v. 28, n. 1, p. 5-36, 2011.

SEN, Amartya. Equality of what? Tanner lecture on human values. Tanner Lectures. Salt Lake City, UT: Stanford Universty, 1979.

SHLENS, Jonathon. Notes on Kullback-Leibler divergence and likelihood. arXivpreprint arXiv:1404, 2000, 2014.

SILVERMAN, Bernard W. Density estimation for statistic and data analysis. Londres: CRC Press, 1986.

SOARES, José Francisco. Measuring cognitive achievement gaps and inequalities: the case of Brazil. International Journal of Educational Research, v. 45 , n. 3, p. 176-187, 2006. 
SOARES, José Francisco. Índice de Desenvolvimento da Educação de São Paulo - IDESP. São Paulo Perspectiva, São Paulo, v. 23, n. 1, p. 29-41, 2009.

SOARES, José Francisco; ALVES, Maria Teresa Gonzaga; XAVIER, Flávia Pereira. Effects of Brazilian schools on student learning. Assessment in Education. Principles, Policy \& Practice, v. 23, n. 1, p. 75-97, 2016.

SOARES, José Francisco; MAROTTA, Luana Castro de Souza; DELGADO, Victor Maia Senna. Measuring the quality and equity of basic education systems. In: ISA WORLD CONGRESS OF SOCIOLOGY, 17., 2010, Sweden. Proceedings... Sweden, 2010.

THOMAS, Vinod; WANG, Yan; FAN, Xibo. Measuring education in equality: Gini coefficient of education. Washington, DC: World Bank, 2001.

TODOS PELA EDUCAÇÃO. De olho nas metas: primeiro relatório de acompanhamento das 5 metas do movimento todos pela educação. Comissão técnica do compromisso Todos pela Educação. São Paulo, 2008. Disponível em: <http://www.todospelaeducacao.org.br/|arquivos/ biblioteca/1a60588b-054d-4422-9a4f-0f009d7b2039.pdf>. Acesso em: 19 set. 2016.

JOSÉ FRANCISCO SOARES

Professor do Programa de Pós-graduação em Educação da Faculdade de Educação (FAE) da Universidade Federal de Minas Gerais (UFMG), Belo Horizonte, Minas Gerais, Brasil francisco-soares@ufmg.br

VICTOR MAIA SENNA DELGADO

Professor do Departamento de Ciências Econômicas da Universidade Federal de Ouro Preto (Ufop), Ouro Preto, Minas Gerais, Brasil vic_senn@yahoo.com.br 
